\title{
MICROWAVE IMAGING BY ELASTIC DEFORMATION
}

\author{
HABIB AMMARI*, YVES CAPDEBOSCQ ${ }^{\dagger}$, FRÉDÉRIC DE GOURNAY $^{\ddagger}$, ANNA $^{*}$ \\ ROZANOVA-PIERRAT ${ }^{\S}$, AND FAOUZI TRIKI $\uparrow$
}

\begin{abstract}
In this paper, we show that using microwave measurements at different frequencies and ultrasound localized perturbations to create local changes in the medium it is possible to extend the method developed by Ammari et al. in [3] to problems in the form

$$
\left\{\begin{array}{l}
\nabla \cdot(a \nabla u)+k^{2} q u=0 \quad \text { in } \Omega \\
u=\varphi \text { on } \partial \Omega
\end{array}\right.
$$

and to reconstruct reliably both the real-valued functions $a$ and $q$ from the internal energies $a|\nabla u|^{2}$ and $q|u|^{2}$.
\end{abstract}

Key words. hybrid imaging, expansion methods, microwave imaging, elastic perturbation, resolution enhancement, explicit inversion formula, optimal control

AMS subject classifications. 31B20, 35B37, 35L05

1. Introduction. The aim of this paper is to develop new mathematical tools and inversion methods to address emerging modalities which are of great current interest in the biomedical imaging community and pose challenging mathematical and numerical problems. We want to extend the hybrid approach for conductivity imaging developed in [3] to the microwave regime.

Emerging imaging modalities are based on a multi-wave concept. Different physical types of waves are combined into one tomographic process to alleviate deficiencies of each separate type of waves, while combining their strengths. Multi-wave systems are capable of high-resolution and high-contrast imaging [1]. A few particular examples of emerging modalities are of great current interest in the biomedical imaging community and will be investigated in detail: magnetic resonance electrical impedance tomography (MREIT) [24, 29], [28], magnetic resonance elastography (MRE) [7], impedance-acoustic tomography [21], photo-acoustic [33, 25, 4] and acousto-optic imaging [14], magneto-acoustic imaging [6], and vibro-acoustography [19].

One way to combine waves is through controlled perturbations. By non intrusive methods, controlled perturbations inside a domain of interest, e.g., a human body, can be created. As first shown in [3], this allows one to reconstruct the unperturbed medium very accurately by using a standard medical imaging technique, which in the absence of these controlled perturbations, provides very poor resolution.

In electrical impedance tomography (EIT), it is well known that the reconstruction of the conductivity from boundary measurements is a very ill-conditioned problem. This drawback has limited its use so far to anomaly detection $[10,11,26]$. In

*Department of Mathematics and Applications, Ecole Normale Supérieure, 45 Rue d'Ulm, 75005 Paris, France (habib.ammari@ens.fr).

†Mathematical Institute 24-29 St Giles' Oxford OX1 3LB, UK (capdeboscq@maths.ox.ac.uk).

‡Laboratoire de Mathématique, Université de Versailles Saint-Quentin-En-Yvelines, Bâtiment Fermat 45, avenue des États-Unis, F-78035 Versailles cedex, France (gournay@math.uvsq.fr).

$\S$ Laboratoire de Mathématiques Appliquées aux Systèmes, École Centrale Paris, Grande Voie des Vignes, 92295 Châtenay-Malabry Cedex, France (anna.rozanova-pierrat@ecp.fr).

『Laboratoire Jean Kuntzmann, Université Joseph Fourier, BP 53, 38041, Grenoble Cedex 9, France (triki.faouzi@imag.fr). 
the recent work by Ammari et al. [3], it was shown that combining these measurements with simultaneous localized ultrasonic perturbations allows to recover the conductivity with good resolution. In fact, we change the EIT problem by the problem of reconstructing the conductivity distribution from the internal electrical energy. The purpose of this paper is to show that such an approach can be generalized successfully to the microwave regime, where the conductivity equation is replaced by a Helmholtz equation and the problem is to reconstruct both the permittivity and the permeability of the medium from internal electromagnetic energies. Recently, microwave imaging has interested many researchers, specially for breast cancer imaging. Microwave imaging is anticipated to be sensitive (detect most tumors in the breast) and specific (specify whether a tumor is malignant or benign). See, for instance, $[17,20,23,27]$.

The core idea of the hybrid method proposed in this paper is to take microwave boundary measurements while perturbing the medium with ultrasound waves focalized on regions of small diameter inside the object. Small-volume asymptotic expansions relate the the difference between the perturbed and unperturbed boundary measurements to pointwise values of electromagnetic energies at the center of the perturbed zone. In contrast to the EIT problem, these energies may vanish within the domain. To overcome this fundamental difficulty, we use many frequencies and many boundary data. We provide an efficient optimization algorithm to solve the reconstruction problem with good resolution in a stable way. We show that by using internal measurements it is possible to significantly overcome the classical Rayleigh resolution limit in microwave imaging. See, for instance, $[1,15]$. As highlighted in [3], the resolution is of order the size of the focal spot of the ultrasound perturbation.

The paper is organized as follows. In Section 2, we recall the effect of a small localized change of the permittivity and the permeability on the microwave boundary measurements. Section 3 is devoted to the derivations of exact reconstruction formulas. Section 4 is to present our reconstruction algorithm which is based on a minimization approach. The initial guesses are constructed using the exact reconstruction formulas of Section 3. The paper ends with a short discussion.

2. Expansion formulas. Let $\Omega$ be a smooth bounded domain in $\mathbb{R}^{2}$. We define the Banach spaces $W^{1, p}(\Omega), 1<p \leq+\infty$, by

$$
W^{1, p}(\Omega)=\left\{u \in L^{p}(\Omega), \nabla u \in L^{p}(\Omega)\right\},
$$

where $\nabla u$ is interpreted as a distribution, and $L^{p}(\Omega)$ is defined in the usual way. The case $p=2$ is special, since the space $W^{1,2}(\Omega)$ is a Hilbert space under the scalar product

$$
(u, v)=\int_{\Omega} u v+\int_{\Omega} \nabla u \cdot \nabla v .
$$

It is also known that the trace operator $\left.u \mapsto u\right|_{\partial \Omega}$ is a bounded linear surjective operator from $W^{1,2}(\Omega)$ into $W_{\frac{1}{2}}^{2}(\partial \Omega)$, where $\varphi \in W_{\frac{1}{2}}^{2}(\partial \Omega)$ if and only if $\varphi \in L^{2}(\partial \Omega)$ and

$$
\int_{\partial \Omega} \int_{\partial \Omega} \frac{|\varphi(x)-\varphi(y)|^{2}}{|x-y|^{2}} d \sigma(x) d \sigma(y)<+\infty .
$$

Let $a \in \mathcal{C}^{1}(\Omega)$ and $q \in \mathcal{C}^{0}(\Omega)$ be two scalar real-valued functions. We also assume that $a$ and $q$ are such that $0<c_{0}<a, q<C_{0}$. For $\varphi \in W_{\frac{1}{2}}^{1,2}(\partial \Omega)$, let 
$u[k, \varphi] \in W^{1,2}(\Omega)$ be such that

$$
\left\{\begin{array}{l}
\nabla \cdot(a \nabla u[k, \varphi])+k^{2} q u[k, \varphi]=0 \quad \text { in } \Omega \\
u=\varphi \quad \text { on } \partial \Omega .
\end{array}\right.
$$

Here $k$ is the angular frequency and $a$ and $q$ are the electromagnetic parameters. In the transverse magnetic case, Maxwell's equations can be reduced to (2.1) with $u$ being the electric field, $q$ the electric permittivity and $a$ the inverse of the magnetic permeability. In this paper, we consider the general case where $a$ is non constant. But all the results and the methods of the paper remain valid in the constant case. The well-posedness of problem (2.1) requires that $k^{2}$ must not be an eigenvalue of the problem

$$
\left\{\begin{array}{l}
-\nabla \cdot(a \nabla u)=k^{2} q u \quad \text { in } \Omega \\
u=0 \quad \text { on } \partial \Omega .
\end{array}\right.
$$

It is well known that this problem admits a countable number of eigenvalues with no accumulation point and that each eigenvalue has a finite multiplicity. We will assume that $k$ does not correspond to any eigenvalue of (2.2).

The generalization of the imaging method introduced in [3] is the following. A frequency $k$ and a source field pattern $\varphi$ being fixed, we measure the field $u[k, \varphi]$, solution of (2.1) on $\partial \Omega$.

Assume now that ultrasonic waves are focalized around a point $z \in \Omega$, creating a local change in the physical parameters of the medium. Suppose that this deformation affects $a$ and $q$ linearly with respect to the amplitude of the ultrasonic signal. Such an assumption is reasonable if the amplitude is not too large. Thus, when the electric potential is measured while the ultrasonic perturbation is enforced, the equation for the electric field is

$$
\left\{\begin{array}{l}
\nabla \cdot\left(a_{\omega} \nabla u_{\omega}\right)+k^{2} q_{\omega} u_{\omega}=0 \quad \text { in } \Omega \\
u_{\omega}=\varphi \text { on } \partial \Omega
\end{array}\right.
$$

with

$$
\left\{\begin{array}{l}
a_{\omega}=a+1_{\omega}\left(a_{1} \alpha-a\right) \\
q_{\omega}=q+1_{\omega}\left(q_{1} \alpha-q\right)
\end{array}\right.
$$

where $\alpha$ is the amplitude of the ultrasonic perturbation and $1_{\omega}$ is the indicator function of the small zone $\omega$ where the perturbation is focalized.

The analysis of the change of the Dirichlet-to-Neumann map as a result of electromagnetic perturbation of small volume is now classical, see $[8,9,12,32]$. The signature of the perturbations on boundary measurements can be measured by the change of energy on the boundary, namely

$$
\begin{aligned}
\int_{\partial \Omega} \frac{\partial}{\partial \nu}\left(u_{\omega}[k, \varphi]-u[k, \varphi]\right) \bar{\varphi} d \sigma= & M\left(\frac{\alpha a_{1}(z)}{a(z)}, \omega\right)\left(\alpha a_{1}(z)-a(z)\right) \nabla u[k, \varphi](z) \cdot \nabla u[k, \varphi](z) \\
& +k^{2}|\omega|\left(\alpha q_{1}(z)-q(z)\right)(u[k, \varphi](z))^{2}+o(|\omega|),
\end{aligned}
$$

where $\partial / \partial \nu$ denotes the normal derivative on $\partial \Omega, z$ is the center of $\omega$, and $M$ is the polarization tensor associated with $\omega$ and the contrast $\alpha a_{1}(z) / a(z)$. Assuming the perturbed region $\omega$ to be a disk, the polarization tensor is given by

$$
M\left(\frac{\alpha a_{1}(z)}{a(z)}, \omega\right)=|\omega| \frac{2 a(z)}{\alpha a_{1}(z)+a(z)} I_{2},
$$


where $I_{2}$ is the $2 \times 2$ identity matrix [9]. Therefore, for a localized perturbation focused at a point $z$, we read the following data (rescaled by the volume $|\omega|$ )

$$
D_{z}(\alpha)=2 a|\nabla u[k, \varphi](z)|^{2} \frac{\alpha \frac{a_{1}(z)}{a(z)}-1}{\alpha \frac{a_{1}(z)}{a(z)}+1}+k^{2} q(z)|u[k, \varphi](z)|^{2}\left(\alpha \frac{q_{1}(z)}{q(z)}-1\right) .
$$

The parameters $\left(a_{1} / a\right)(z)$ and $\left(q_{1} / q\right)(z)$ are unknown, but the amplitude $\alpha$ is known. By linear algebra, one can prove from (2.4) that if $|\nabla u[k, \varphi](z)| \gg|\omega|$ and $|u[k, \varphi](z)| \gg|\omega|$ and the data $D_{z}$ is known for 4 distinct values of $\alpha$, chosen independently of $a$ and $q$, then, one can recover the electromagnetic energies

$$
E[k, \varphi](z):=a(z)|\nabla u[k, \varphi](z)|^{2},
$$

and

$$
e[k, \varphi](z):=q(z)|u[k, \varphi](z)|^{2} .
$$

Since [24], internal energies have been used to provide efficient imaging procedures, see $[3,6,5,14,21,28]$.

At this point, one can respectively substitute $a$ and $q$ by $E[k, \varphi] /|\nabla u[k, \varphi]|^{2}$ and $e[k, \varphi] /|u[k, \varphi]|^{2}$ to arrive at the nonlinear partial differential equation

$$
\left\{\begin{array}{l}
\nabla \cdot\left(\frac{E[k, \varphi]}{|\nabla u[k, \varphi]|^{2}} \nabla u[k, \varphi]\right)+k^{2} \frac{e[k, \varphi]}{|u[k, \varphi]|^{2}} u[k, \varphi]=0 \quad \text { in } \Omega \\
u=\varphi \text { on } \partial \Omega .
\end{array}\right.
$$

Based on the nonlinear direct formulation (2.5), an iterative scheme similar to the one introduced in [2] can be derived. However, as noticed in [16], an optimal control approach is more efficient for reconstructing the parameters than the nonlinear based direct formulation (2.5), specially when the data is available only on a subset of the background medium $\Omega$.

3. Exact reconstruction formulas. In this section, we give explicit formulas for reconstructing $a$ and $q$. Unfortunately, these formulas involve derivatives of the data and then can only be used to construct a good initial guess.

We first note that in contrast to the conductivity case (when $k=0$ ), the solution $u_{i}$ may vanish within the domain and the same for its gradient. Measurements where $u_{i}$ or its gradient nearly vanish are not significant, since measurement errors become dominant. It is therefore sensible to use the measurements in areas of confidence, that is, where they are sufficiently large. We will call such an area $D$. For a given focalization, two parameters are available to the practitioner. Both the source field pattern $\varphi$ and the frequency $k$ can be changed. Changing either parameter modifies the zero level-set of $E[k, \varphi]$ or $e[k, \varphi]$. Varying the focalization point, we are then able to recover this localized internal data everywhere inside the domain $D$.

We need the following definition.

Definition 3.1. A set of $N \geq 2$ pair of parameters

$$
\left(k_{i}, \varphi_{i}\right)_{1 \leq i \leq N} \in\left((0, \infty) \times W_{\frac{1}{2}}^{1,2}(\partial \Omega)\right)^{N},
$$

defines a proper set of measurements $\mathcal{M}(D, \alpha, \beta)$ for $D \subset \Omega$ if there exist two positive constants $\alpha>0$ and $\beta>0$ such that 
(i) For all $z \in D$,

$$
1 \leq \sum_{i=1}^{N} e\left[k_{i}, \varphi_{i}\right](z) \leq \beta
$$

(ii) For all $z \in D$,

$$
1 \leq \sum_{i=1}^{N} E\left[k_{i}, \varphi_{i}\right](z) \leq \beta
$$

(iii) For every $z \in D$ there exist $i, j$ (which may depend on $z$ ) such that

$$
\left|\operatorname{det}\left(\nabla u\left[k_{i}, \varphi_{i}\right](z), \nabla u\left[k_{j}, \varphi_{j}\right](z)\right)\right| \geq \alpha,
$$

where det denotes the determinant.

A proper set of measurements $\mathcal{M}(D, \alpha, \beta)$ is a complete set of measurements if additionally, $N \geq 3, e\left[k_{i}, \varphi_{i}\right] \in W^{1, \infty}(D)$ and

(iv) For every $z \in D$ there exist $i, j, l$ (which may depend on $z$ ) such that

$$
\left|\operatorname{det}\left(\begin{array}{c|c|c}
\nabla u\left[k_{i}, \varphi_{i}\right](z) & \nabla u\left[k_{j}, \varphi_{j}\right](z) & \nabla u\left[k_{l}, \varphi_{l}\right](z) \\
u\left[k_{i}, \varphi_{i}\right](z) & u\left[k_{j}, \varphi_{j}\right](z) & u\left[k_{l}, \varphi_{l}\right](z)
\end{array}\right)\right| \geq \alpha^{3 / 2} .
$$

The first two requirements (i) and (ii) are necessary, for if they are not satisfied, data cannot be recovered at each point. The constant 1 is arbitrary, and is not a constraint, as it is always possible to increase the amplitude of the source field patterns $\varphi_{i}$ by a multiplicative constant. The constraint (iii) implies that there are at least two independent measurements for the gradient term at each point. The constraint (iv) implies that there are at least three pairwise independent measurements. Note that we do not require that the same solution $u\left[k_{i}, \varphi_{i}\right]$ satisfies both (i) and (ii). This makes the constraint on data to be a proper set of measurements quite mild. In any case, there always exist proper sets of measurements. Using advanced results on the so-called geometric optic solutions [30,13], one can show that when $N=2$, the much more stringent requirement that all three conditions are satisfied by the a single (complex) pair of data is satisfied for some carefully selected $\varphi$. See, for instance, [31].

We use the notation that if $M$ (resp. $V$ ) is a $N \times N$ matrix (resp. $N \times 1$ vector) valued function,

$$
|M|_{2}^{2}=\sum_{i j} M_{i j}^{2},|\nabla M|_{2}^{2}=\sum_{i j}\left|\nabla M_{i j}\right|^{2},|V|_{2}^{2}=\sum_{i=1}^{N} V_{i}^{2} \text {, and }|\nabla V|_{2}^{2}=\sum_{i=1}^{N}\left|\nabla V_{i}\right|^{2} .
$$

The reconstruction problem then becomes:

Problem. Assume that $N \geq 2$, and that

$$
\left(k_{i}, \varphi_{i}\right)_{1 \leq i \leq N} \in\left((0, \infty) \times W_{\frac{1}{2}}^{1,2}(\partial \Omega)\right)^{N},
$$

is a $\mathcal{M}(D, \alpha, \beta)$ proper set of measurements for $D \subset \Omega$. Suppose the matrix-valued functions $e$ and $E$ are given by

$$
E_{i j}=a(z) \nabla u\left[k_{i}, \varphi_{i}\right] \cdot \nabla u\left[k_{j}, \varphi_{j}\right],
$$

and

$$
e_{i j}=q(z) u\left[k_{i}, \varphi_{i}\right] \cdot u\left[k_{j}, \varphi_{j}\right] .
$$

Find $a$ and $q$ in $D$. 
Remark 3.2. Note that using (2.3) and (2.4) the 'polarized' data $E_{i j}$ and $e_{i j}$ for $i \neq j$ is available without additional measurements, thanks to the bilinear structure of the asymptotic formula (2.3). In fact, we have

$$
\begin{aligned}
\int_{\partial \Omega} \frac{\partial}{\partial \nu}\left(u_{\omega}[k, \varphi]-u[k, \varphi]\right) \bar{\psi} d \sigma= & M\left(\frac{\alpha a_{1}(z)}{a(z)}, \omega\right)\left(\alpha a_{1}(z)-a(z)\right) \nabla u[k, \varphi](z) \cdot \nabla u[k, \psi](z) \\
& +k^{2}|\omega|\left(\alpha q_{1}(z)-q(z)\right) u[k, \varphi](z) u[k, \psi](z)+o(|\omega|) .
\end{aligned}
$$

The following results hold. They will be used to construct an initial guess for $a$ and $q$.

Proposition 3.3. Assume that $N \geq 2$, and that

$$
\left(k_{i}, \varphi_{i}\right)_{1 \leq i \leq N} \in\left((0, \infty) \times W_{\frac{1}{2}}^{1,2}(\partial \Omega)\right)^{N}
$$

is a $\mathcal{M}(D, \alpha, \beta)$ proper set of measurements for $D \subset \Omega$. Let $P_{U}$ be the projection in $\mathbb{R}^{N}$ on the unit vector $U$ given by

$$
U_{i}=\frac{u\left[k_{i}, \varphi_{i}\right]}{\sqrt{\sum_{n=1}^{N} u\left[k_{n}, \varphi_{n}\right]^{2}}}, \quad \forall x \in D, 1 \leq i \leq N .
$$

Then $P_{U}$ is given in terms of the data by

$$
\left(P_{U}\right)_{i j}=\frac{e_{i j}}{\operatorname{tr}(e)}
$$

where tr denotes the trace. Furthermore, $P_{U} \in W^{1, \infty}(D)$ and satisfies

$$
\frac{1}{2}\left|\nabla P_{U}\right|_{2}^{2}=\frac{q}{a} \frac{\operatorname{tr}\left(E-P_{U} E\right)}{\operatorname{tr}(e)}
$$

which allows to determine $q / a$, as

$$
\operatorname{tr}\left(E-P_{U} E\right) \geq \operatorname{tr}(E) \frac{\alpha^{4}}{\beta^{4}}>0 .
$$

Moreover, the following proposition gives an explicit formula to determine $q$ up to a multiplicative constant.

Proposition 3.4. Under the same assumptions as those in Proposition 3.3, suppose that $\operatorname{tr}(e) \in W^{1, \infty}(D)$. We have

$$
\frac{\partial_{x_{k}} q}{q}=\frac{\partial_{x_{k}} \operatorname{tr}(e)}{\operatorname{tr}(e)}-2 \lambda_{k}, \quad k=1,2,
$$

where $\lambda_{1}$ and $\lambda_{2}$ satisfy

$$
\lambda_{1}^{2}+\lambda_{2}^{2}=\frac{q}{a} \frac{\operatorname{tr}\left(P_{U} E\right)}{\operatorname{tr}(e)}
$$

and are determined by the linear system

$$
M\left(\begin{array}{c}
\lambda_{1} \\
\lambda_{2}
\end{array}\right)=B
$$


with

$$
M=\left(\begin{array}{cc}
\left|\partial_{x_{1}} U\right|^{2} & \partial_{x_{1}} U \cdot \partial_{x_{2}} U \\
\partial_{x_{1}} U \cdot \partial_{x_{2}} U & \left|\partial_{x_{2}} U\right|^{2}
\end{array}\right) \text { and } B=\frac{q}{\operatorname{atr}(e)}\left(\begin{array}{c}
E U \cdot \partial x_{1} U \\
E U \cdot \partial x_{2} U
\end{array}\right) .
$$

For all $N \geq 2$, we have by assumption that

$$
|\nabla U|_{2}>\frac{\alpha^{2}}{\beta^{2}}
$$

and thus, $\operatorname{rank}(M) \geq 1$. If $N=2$, then $\operatorname{rank}(M)=1$. If $N \geq 3$ and $\mathcal{M}(D, \alpha, \beta)$ is a complete set of measurements, then

$$
|\operatorname{det}(M)|>\alpha^{3} \frac{q^{3}}{\operatorname{tr}(e)^{3}},
$$

which shows the invertibility of the linear system (3.5).

Before proving Propositions 3.3 and 3.4, we shall make a few remarks.

Remark 3.5. Note that in all cases, (3.4) and (3.5) leave at most 2 choices $\nabla q(z) / q$ for each $z$. So provided for example that $\nabla q$ is continuous, and that $\nabla q$ and $\nabla^{2} q$ have no common roots, $\nabla q$ is globally determined in $D$ as being one of the two possibilities. If $q$ is known by other means on a subset of $D$, then $q$ is also completely determined in the case of a proper (and not complete) set of measurements.

Remark 3.6. It is also worth noticing that (3.1) and (3.3) do not depend on the number $N \geq 3$ of measurements. From the numerical point of view, adding more measurements even though they are of lower signal-to-noise ratio (SNR) would increase the overall quality of the reconstruction.

Remark 3.7. Finally, we shall note that the exact reconstruction formulas given in Propositions 3.3 and 3.4 are not valid when a and/or $q$ are complex.

Proof of Proposition 3.3. Let $T:=e_{i j} / \operatorname{tr}(e)$. Since we have an $\mathcal{M}(D, \alpha, \beta)$ proper set of measurements, $\operatorname{tr}(e) \geq 1$ in $D$, thus $T_{i j}$ is well defined. Furthermore,

$$
T_{i j}=\frac{u\left[k_{i}, \varphi_{i}\right] u\left[k_{j}, \varphi_{j}\right]}{\sum_{n=1}^{N} u\left[k_{n}, \varphi_{n}\right]^{2}}=U_{i} U_{j}
$$

thus $T=P_{U}$, as announced. Differentiating this formula, we obtain

$$
\frac{1}{2}\left|\nabla P_{U}\right|_{2}^{2}=|\nabla U|_{2}^{2}|U|_{2}^{2}+\left|\sum_{i=1}^{N} U_{i} \nabla U_{i}\right|^{2}=|\nabla U|_{2}^{2},
$$

since $\sum_{i=1}^{N} U_{i}^{2}=1$. We compute that

$$
\begin{aligned}
\left(\sum_{n=1}^{N} u\left[k_{n}, \varphi_{n}\right]^{2}\right)^{2}|\nabla U|_{2}^{2} & =\left(\sum_{n=1}^{N} u\left[k_{n}, \varphi_{n}\right]^{2}\right) \sum_{p=1}^{N}\left|\nabla u\left[k_{p}, \varphi_{p}\right]\right|^{2} \\
& -\sum_{p=1}^{N} \sum_{n=1}^{N} \nabla u\left[k_{n}, \varphi_{n}\right] \cdot \nabla u\left[k_{p}, \varphi_{p}\right] u\left[k_{n}, \varphi_{n}\right] u\left[k_{p}, \varphi_{p}\right],
\end{aligned}
$$

which can be written also

$$
\frac{a}{q}|\nabla U|_{2}^{2}=\frac{1}{\operatorname{tr}(e)}\left(\operatorname{tr}(E)-\operatorname{tr}\left(P_{U} E\right)\right),
$$


and we have obtained (3.1). Now note that $E$ can be written

$$
E=\operatorname{tr}(E)\left(\frac{n_{1}^{2}}{n_{1}^{2}+n_{2}^{2}} P_{U, 1}+\frac{n_{2}^{2}}{n_{1}^{2}+n_{2}^{2}} P_{U, 2}\right),
$$

where we wrote

$$
\left(U_{, k}\right)_{i}:=\frac{\partial_{x_{k}} u\left[k_{i}, \varphi_{i}\right]}{n_{k}}, \text { and } n_{k}:=\sqrt{\sum_{i=1}^{N}\left(\partial_{x_{k}} u\left[k_{i}, \varphi_{i}\right]\right)^{2}} \text { for } k=1,2 .
$$

Note that $n_{k}, U_{, k} \neq 0$. Therefore, it is immediate that

$$
\operatorname{tr}\left(P_{U} E\right)=\operatorname{tr}(E)\left(\frac{n_{1}^{2}}{n_{1}^{2}+n_{2}^{2}}\left(U \cdot U_{, 1}\right)^{2}+\frac{n_{2}^{2}}{n_{1}^{2}+n_{2}^{2}}\left(U \cdot U_{, 2}\right)^{2}\right)
$$

Note that $U_{, 1}, U_{, 2}$ are well defined. Indeed, Since we have $\mathcal{M}(D, \alpha, \beta)$ a proper set of measurements, there exists $i, j$ such that

$$
\left|\left(\begin{array}{c}
\partial_{x_{1}} u\left[k_{i}, \varphi_{i}\right] \\
\partial_{x_{1}} u\left[k_{j}, \varphi_{j}\right]
\end{array}\right) \cdot\left(\begin{array}{r}
-\partial_{x_{2}} u\left[k_{j}, \varphi_{j}\right] \\
\partial_{x_{2}} u\left[k_{i}, \varphi_{i}\right]
\end{array}\right)\right| \geq \alpha
$$

so in particular

$$
\frac{n_{1} n_{2}}{n_{1}^{2}+n_{2}^{2}} \geq \frac{\alpha}{\beta}
$$

Writing that $U_{, 1}=\left(U_{, 1} \cdot U_{, 2}\right) U_{, 2}+C U_{, 2}^{\perp}+D$, with $U_{, 2}^{\perp}$ having only two non zero components, $-\partial_{x_{2}} u\left[k_{j}, \varphi_{j}\right]$ in position $i$ and $\partial_{x_{2}} u\left[k_{i}, \varphi_{i}^{\prime}\right]$ in position $j$, and $D$ being the remainder, orthogonal to both $U_{, 2}$ and $U_{, 2}^{\frac{1}{2}}$, we obtain

$$
1 \geq\left(U_{, 1} \cdot U_{, 2}\right)^{2}+\frac{\alpha^{2}}{n_{1}^{2} n_{2}^{2}},
$$

or in other words,

$$
\left(U_{, 1} \cdot U_{, 2}\right)^{2} \leq 1-\frac{\alpha^{2}\left(n_{1}^{2}+n_{2}^{2}\right)^{2}}{\beta^{2} n_{1}^{2} n_{2}^{2}} \leq 1-4 \frac{\alpha^{2}}{\beta^{2}} .
$$

Decomposing $U$ in the orthogonal basis of $\mathbb{R}^{N}$ starting by $U_{, 1}, U_{, 2}-\left(U_{, 1} \cdot U_{, 2}\right) U_{, 1}$, and using the fact that $U$ is of unit norm, we obtain

$$
1-\left(U_{, 1} \cdot U_{, 2}\right)^{2} \geq\left(U \cdot U_{, 1}\right)^{2}+\left(U \cdot U_{, 2}\right)^{2}-2\left(U_{, 1} \cdot U_{, 2}\right)\left(U \cdot U_{, 1}\right)\left(U \cdot U_{, 2}\right),
$$

and therefore,

$$
\left(U \cdot U_{, 1}\right)^{2}+\left(U \cdot U_{, 2}\right)^{2} \leq 1+\left|U_{, 1} \cdot U_{, 2}\right| \leq 1+\sqrt{1-4 \frac{\alpha^{2}}{\beta^{2}}} \leq 2\left(1-\frac{\alpha^{2}}{\beta^{2}}\right) .
$$

Combining (3.8), (3.9) and (3.10), and using the trivial bound $\left|U \cdot U_{, k}\right| \leq 1$, we obtain

$$
\begin{aligned}
\operatorname{tr}\left(P_{U} E\right) & \leq \operatorname{tr}(E)\left(\left(1-\frac{\alpha^{2}}{\beta^{2}}\right) \min \left(\frac{n_{1}^{2}}{n_{1}^{2}+n_{2}^{2}}, \frac{n_{2}^{2}}{n_{1}^{2}+n_{2}^{2}}\right)+\max \left(\frac{n_{1}^{2}}{n_{1}^{2}+n_{2}^{2}}, \frac{n_{2}^{2}}{n_{1}^{2}+n_{2}^{2}}\right)\right) \\
& \leq \operatorname{tr}(E)\left(1-\frac{\alpha^{4}}{\beta^{4}}\right),
\end{aligned}
$$


and hence (3.2) follows.

Proof of Proposition 3.4. Differentiating the formula for $\operatorname{tr}(e)$, we obtain

$$
\frac{\partial_{x_{k}} \operatorname{tr}(e)}{\operatorname{tr}(e)}=\frac{\partial_{x_{k}} q}{q}+2 \lambda_{k}, \quad k=1,2,
$$

where, using the same notation as in Proposition 3.3,

$$
\lambda_{k}=\frac{1}{\sum_{i=1}^{N} u\left[k_{i}, \varphi_{i}\right]^{2}} \sum_{n=1}^{N} u\left[k_{n}, \varphi_{n}\right] \partial_{x_{k}} u\left[k_{n}, \varphi_{n}\right]=\frac{n_{k}}{n_{0}} U \cdot U_{, k},
$$

with the additional notation that

$$
n_{0}:=\sqrt{\sum_{i=1}^{N}\left(u\left[k_{i}, \varphi_{i}\right]\right)^{2}}
$$

Differentiating $U$, we find

$$
\partial_{x_{k}} U=\frac{n_{k}}{n_{0}}\left(I_{N}-P_{U}\right) U_{, k},
$$

where $I_{N}$ is the $N \times N$ identity matrix. Writing the matrix $E$ in the form

$$
E=\operatorname{tr}(E) \sum_{k=1}^{2} \frac{n_{k}^{2}}{n_{1}^{2}+n_{2}^{2}} P_{U_{, k}}
$$

we compute that

$$
E U=\operatorname{tr}(E) \sum_{k=1}^{2} \frac{n_{k}^{2}}{n_{1}^{2}+n_{2}^{2}}\left(U_{, k} \cdot U\right) U_{, k}=a \sum_{k=1}^{2} n_{k} n_{0} \lambda_{k} U_{, k} .
$$

Testing (3.12) against $U$, we obtain

$$
E U \cdot U=a \sum_{k=1}^{2} n_{k} n_{0} \lambda_{k} U_{, k} \cdot u=a \sum_{k=1}^{2} n_{0}^{2} \lambda_{k}^{2},
$$

which is (3.4). Alternatively, testing (3.12) against $\partial_{x_{l}} U$ gives, using (3.11),

$$
\begin{aligned}
E U \cdot \partial_{x_{l}} U & =a \sum_{k=1}^{2} n_{k} n_{l} \lambda_{k} U_{, k} \cdot\left(I_{N}-P_{U}\right) U_{, l} \\
& =a \sum_{k=1}^{2} n_{k} n_{l} \lambda_{k}\left(I_{N}-P_{U}\right) U_{, k} \cdot\left(I_{N}-P_{U}\right) U_{, l} \\
& =a \sum_{k=1}^{2} n_{0}^{2} \lambda_{k} \partial_{x_{k}} U \cdot \partial_{x_{l}} U \\
& =\frac{a}{q} \operatorname{tr}(e) \sum_{k=1}^{2} \lambda_{k} \partial_{x_{k}} U \cdot \partial_{x_{l}} U
\end{aligned}
$$


which is the desired $2 \times 2$ system given by (3.5). Note that since $|U|_{2}^{2}=1$, we have $\partial_{x_{k}} U \cdot U=0$. Therefore, if $U$ has only two components, $\partial_{x_{1}} U$ and $\partial_{x_{2}} U$ are necessarily colinear, and system (3.5) is degenerate.

However, it is never a zero matrix. Indeed,

$$
|\nabla U|_{2}^{2}=\frac{1}{2}\left|\nabla P_{U}\right|_{2}^{2}
$$

and therefore thanks to Proposition 3.3,

$$
|\nabla U|_{2}>\frac{\alpha^{2}}{\beta^{2}}
$$

Suppose now that $\mathcal{M}(D, \alpha, \beta)$ is a complete set of measurements. To fix ideas, let us say that

$$
D:=\left|\operatorname{det}\left(\begin{array}{ccc}
\partial_{x_{1}} u\left[k_{1}, \varphi_{1}\right] & \partial_{x_{2}} u\left[k_{1}, \varphi_{1}\right] & u\left[k_{1}, \varphi_{1}\right] \\
\partial_{x_{1}} u\left[k_{2}, \varphi_{2}\right] & \partial_{x_{2}} u\left[k_{2}, \varphi_{2}\right] & u\left[k_{2}, \varphi_{2}\right] \\
\partial_{x_{1}} u\left[k_{3}, \varphi_{3}\right] & \partial_{x_{2}} u\left[k_{3}, \varphi_{3}\right] & u\left[k_{3}, \varphi_{3}\right]
\end{array}\right)\right| \geq \alpha^{3 / 2}
$$

that is, $i=1, j=2, l=3$. Note that

$$
D=\left|\operatorname{det}\left(U_{, 1} n_{1}, U_{, 2} n_{2}, U n_{0}, e_{4}, \ldots, e_{N}\right)\right|,
$$

where $e_{i t h}$ is the $i$-th canonical basis element of $\mathbb{R}^{N}$. Consequently,

$$
\begin{aligned}
D & =n_{0} n_{1} n_{2}\left|\operatorname{det}\left(U_{, 1}, U_{, 2}, U, e_{4}, \ldots, e_{N}\right)\right|, \\
& =n_{0} n_{1} n_{2}\left|\operatorname{det}\left(U_{, 1}-P_{U} U, 1, U_{, 2}-P_{U} U_{, 2}, U, e_{4}, \ldots, e_{N}\right)\right|, \\
& =n_{0}^{3}\left|\operatorname{det}\left(\partial_{x_{1}} U, \partial_{x_{2}} U, U, e_{4}, \ldots, e_{N}\right)\right|, \\
& =n_{0}^{3}\left|\operatorname{det}\left(\partial_{x_{1}} U-\frac{\partial_{x_{1}} U \cdot \partial_{x_{2}} U}{\left|\partial_{x_{2}} U\right|_{2}^{2}} \partial_{x_{2}} U, \partial_{x_{2}} U, U, e_{4}, \ldots, e_{N}\right)\right|, \\
& =n_{0}^{3}\left|\partial_{x_{1}} U-\frac{\partial_{x_{1}} U \cdot \partial_{x_{2}} U}{\left|\partial_{x_{2}} U\right|_{2}^{2}} \partial_{x_{2}} U\right|_{2}\left|\partial_{x_{2}} U\right|_{2} .
\end{aligned}
$$

Now, writing

$$
\left|\partial_{x_{1}} U-\frac{\partial_{x_{1}} U \cdot \partial_{x_{2}} U}{\left|\partial_{x_{2}} U\right|_{2}^{2}} \partial_{x_{2}} U\right|_{2}^{2}\left|\partial_{x_{2}} U\right|_{2}^{2}=\left|\partial_{x_{1}} U\right|_{2}^{2}\left|\partial_{x_{2}} U\right|_{2}^{2}-\left|\partial_{x_{1}} U \cdot \partial_{x_{2}} U\right|^{2},
$$

we obtain that

$$
|\operatorname{det} M| n_{0}^{6} \geq \alpha^{3}
$$

or in other words,

$$
\operatorname{det} M \geq \alpha^{3} \frac{q^{3}}{\operatorname{tr}(e)^{3}}
$$

as desired. 
4. Optimal control algorithm. In this section, we discuss how the scalar coefficients $a$ and $q$ can be recovered in practice from a complete set of measurements $e$ and $E$ in $\Omega$ (see Definition 3.1), provided that $a$ and $q$ are known on a neighborhood of $\partial \Omega$. It is natural to think of a minimization approach, namely,

$$
\text { minimise } J(\gamma, c):=\sum_{i=1}^{N} \int_{\Omega}\left(\left(\gamma\left|\nabla \psi_{i}\right|^{2}\right)^{\frac{1}{2}}-E_{i i^{\frac{1}{2}}}\right)^{2}+\left(\left(c \psi_{i}^{2}\right)^{\frac{1}{2}}-e_{i i^{\frac{1}{2}}}\right)^{2},
$$

where $\psi_{i}$ is the solution of

$$
\left\{\begin{array}{l}
\nabla \cdot\left(\gamma \nabla \psi_{i}\right)+k_{i}^{2} c \psi_{i}=0 \quad \text { in } \Omega \\
\psi_{i}=\varphi_{i} \text { on } \partial \Omega
\end{array}\right.
$$

with $\varphi_{i}, i=1, \ldots, N$, being given boundary source field patterns.

Propositions 3.3 and 3.4 show that, under an appropriate regularity assumption, $J$ admits a unique global minimizer $a$ and $q$. To minimize the quadratic misfit functional $J$, a gradient descent algorithm seems appropriate [18, 22]. It was proved to be very successful when the frequency $k=0$. In [16], the zero frequency case, $k=0$, was considered for both the two- and three-dimensional case. The authors showed that the minimization procedure was very robust: without a good initial guess, the minimization procedure converges to the correct solution.

The situation is dramatically different when $k$ is not close to zero. In order to insure that problem (4.1) is well-posed, $k_{i}$ should not be an eigenvalue of the corresponding homogeneous Dirichlet problem. Enforcing that constraint at every step of the minimization procedure, where $\gamma$ and $c$ are changing, is extremely difficult, and possibly futile. In fact, if the procedure starts from an arbitrary $\gamma$ and $c$, it is unlikely that $k_{i}$ would be located in the same spectral gap for that problem and for the target one. Numerical experiments show that if we start with a randomly chosen initial guess, the iterative procedure quickly stalls near a point where $k_{i}$ is an eigenvalue for (4.1). We therefore need to use the explicit formulas given by Propositions 3.3 and 3.4 to build a good initial guess of the solution.

Our resolution method contains therefore two parts. First, we compute $\gamma_{0}$ and $c_{0}$ following Propositions 3.3 and 3.4. Then we perform a gradient descent on $J$ to improve this initial guess.

Remark 4.1. The reader may wonder why we choose to take $L^{2}$ norm of the square root of the error with respect to the square root of the data. The reason is that the data is known to be in $L^{2}(\Omega)$ so the quadratic difference is in $L^{1}(\Omega)$. Indeed, if one takes an objective functional that resembles

$$
J(\gamma, c)=\sum_{i=1}^{N} \int_{\Omega}\left(\gamma\left|\nabla \psi_{i}\right|^{2}-E_{i i}\right)^{2}+\left(c \psi_{i}^{2}-e_{i i}\right)^{2},
$$

then, one should ensure that $\psi_{i}$ belongs to the space $W^{1,4}(\Omega)$, which is not the natural physical space to work in. On the other hand, even though it requires the natural regularity in $\psi_{i}$, the objective functional

$$
J(\gamma, c):=\left.\sum_{i=1}^{N} \int_{\Omega}|\gamma| \nabla \psi_{i}\right|^{2}-E_{i i}|+| c \psi_{i}^{2}-e_{i i} \mid,
$$

cannot be used since it is not differentiable as soon as a $\left|\nabla \psi_{i}\right|^{2}=E_{i i}$ at some point in $\Omega$. 
4.1. Computation of the initial guess. Proposition 3.3 gives an explicit formula for $q / a$, namely

$$
\frac{1}{2}\left|\nabla P_{U}\right|_{2}^{2}=\frac{q}{a} \frac{\operatorname{tr}\left(E-P_{U} E\right)}{\operatorname{tr}(e)}
$$

where

$$
P_{U}=\frac{e}{\operatorname{tr}(e)}
$$

The implementation is straightforward. Proposition 3.4 gives a formula for the gradient of $q$ in terms of $A, U, e$ and $E$. To compute $U$, we note that it is the range (pointwise in $\Omega$ ) of $P_{U}$, which can thus be obtained by either a power method, or simply, by finding a non-zero column of $P_{U}$. Since $|U|=1$, at least one of the coefficients of $U$ is larger than $1 / \sqrt{N}$, so a simple sorting argument provides $U$. Then, $\ln (q)$ is approximated by $v$, the solution of

$$
-\Delta v=\nabla \cdot(\nabla \ln (\operatorname{tr}(e))-\lambda), \quad v=\ln (q) \text { on } \partial \Omega,
$$

where $\lambda=\left(\lambda_{1}, \lambda_{2}\right)^{T}, T$ denoting the transpose, is calculated as $\lambda=M^{-1} B$, with $M$ and $B$ given by (3.6).

4.2. Computation of the derivative of $J$. We use standard differentiation of the solution of a linear operator with respect to a change of coefficient. Changing $\gamma$ and $c$ by $\gamma+\gamma^{\prime}, c+c^{\prime}$ changes $\psi_{i}$ by $\psi_{i}+\psi_{i}^{\prime}$ where $\psi_{i}^{\prime}$ verifies, at the first order, the equation given by an implicit function theorem

$$
\begin{aligned}
\nabla \cdot\left(\gamma \nabla \psi_{i}^{\prime}\right)+k_{i}^{2} c \psi_{i}^{\prime} & =-\nabla \cdot\left(\gamma^{\prime} \nabla \psi_{i}\right)-k_{i}^{2} c^{\prime} \psi_{i} \quad \text { in } \Omega, \\
\psi_{i}^{\prime} & =0 \quad \text { on } \partial \Omega .
\end{aligned}
$$

Performing usual derivation, $J$ is changed by $J+J^{\prime}$ where $J^{\prime}$, the Fréchet derivative of $J$, verifies

$$
\begin{aligned}
J^{\prime} & =\sum_{i=1}^{N} \int_{\Omega}\left(\sqrt{\gamma}\left|\nabla \psi_{i}\right|-\sqrt{E_{i i}}\right)\left(\frac{\gamma^{\prime}}{\sqrt{\gamma}}\left|\nabla \psi_{i}\right|+2 \sqrt{\gamma} \frac{\nabla \psi_{i}^{\prime} \nabla \psi_{i}}{\left|\nabla \psi_{i}\right|}\right) \\
& +\int_{\Omega}\left(\sqrt{c}\left|\psi_{i}\right|-\sqrt{e_{i i}}\right)\left(\frac{c^{\prime}}{\sqrt{c}}\left|\psi_{i}\right|+2 \sqrt{c} \frac{\psi_{i}^{\prime} \psi_{i}}{\left|\nabla \psi_{i}\right|}\right) .
\end{aligned}
$$

Denoting the errors

$$
\epsilon_{i}(\gamma)=1-\sqrt{\frac{E_{i i}}{a\left|\nabla \psi_{i}\right|^{2}}} \text { and } \epsilon_{i}(c)=1-\sqrt{\frac{e_{i i}}{c\left|\psi_{i}\right|^{2}}},
$$

we have

$$
J^{\prime}=\sum_{i=1}^{N} \int_{\Omega} \epsilon_{i}(\gamma)\left(\gamma^{\prime}\left|\nabla \psi_{i}\right|^{2}+2 \gamma \nabla \psi_{i}^{\prime} \nabla \psi_{i}\right)+\epsilon_{i}(c)\left(c^{\prime}\left|\psi_{i}\right|^{2}+2 c \psi_{i}^{\prime} \psi_{i}\right) .
$$

To remove the terms involving $\psi_{i}^{\prime}$, which are implicitly given by $\gamma^{\prime}$ and $c^{\prime}$, we use the "adjoint method". See, for instance, [22]. The "adjoint method" amounts to compute, for each $i=1, \ldots, N, p_{i}$ as the unique solution in $W^{1,2}(\Omega)$ of

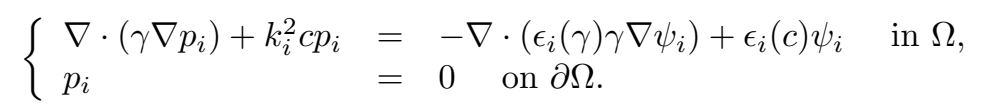


An integration by parts together with the system in $p_{i}$ simplifies the computation of $J^{\prime}$ :

$$
J^{\prime}=\sum_{i=1}^{N} \int_{\Omega} \epsilon_{i}(\gamma) \gamma^{\prime}\left|\nabla \psi_{i}\right|^{2}+\epsilon_{i}(c) c^{\prime}\left|\psi_{i}\right|^{2}-2 \gamma \nabla \psi_{i}^{\prime} \nabla p_{i}+2 c k_{i}^{2} \psi_{i}^{\prime} p_{i}
$$

Using the equation (4.2), defining $\psi_{i}^{\prime}$ and performing an integration by parts, one finds

$$
J^{\prime}=\int_{\Omega} \gamma^{\prime}\left(\sum_{i=1}^{N} \epsilon_{i}(\gamma)\left|\nabla \psi_{i}\right|^{2}+2 \nabla \psi_{i} \nabla p_{i}\right)+c^{\prime}\left(\sum_{i=1}^{N} \epsilon_{i}(c)\left|\psi_{i}\right|^{2}-2 k_{i}^{2} \psi_{i} p_{i}\right) .
$$

The gradient descent algorithm amounts take as descent direction $\gamma^{\prime}$ and $c^{\prime}$ corresponding to

$$
\begin{aligned}
& \gamma^{\prime}=-\sum_{i=1}^{N}\left(\epsilon_{i}(\gamma)\left|\nabla \psi_{i}\right|^{2}+2 \nabla \psi_{i} \nabla p_{i}\right), \\
& c^{\prime}=-\sum_{i=1}^{N}\left(\epsilon_{i}(c)\left|\psi_{i}\right|^{2}-2 k_{i}^{2} \psi_{i} p_{i}\right) .
\end{aligned}
$$

For numerical reasons, we prefer to perform the change of variables $\gamma=e^{\sigma}, c=e^{\mu}$, in order to ensure positivity. In this case, the descent direction is given by

$$
\begin{aligned}
\sigma^{\prime} & =-\frac{1}{\gamma} \sum_{i=1}^{N}\left(\epsilon_{i}(\gamma)\left|\nabla \psi_{i}\right|^{2}+2 \nabla \psi_{i} \nabla p_{i}\right), \\
\mu^{\prime} & =-\frac{1}{c} \sum_{i=1}^{N}\left(k^{2} \epsilon_{i}(c)\left|\psi_{i}\right|^{2}-2 k_{i}^{2} \psi_{i} p_{i}\right) .
\end{aligned}
$$

4.3. Numerical experiments. As a test case, we assume that the domain is a disk of unit radius, where the coefficients $a$ and $q$ are given by

$$
a=\left\{\begin{array}{ll}
2.0 & \text { in } B, \\
1.2 & \text { in } C, \\
2.5 & \text { in } E, \\
1.0 & \text { otherwise }
\end{array} \quad q= \begin{cases}2.0 & \text { in } B, \\
1.8 & \text { in } C, \\
1.2 & \text { in } E \\
1.0 & \text { otherwise }\end{cases}\right.
$$

The set $B$ is the rectangle with diagonal $(0.0,0.4)-(0.3,0.5)$. The set $C$ is the interior area delimited by the curve $t \rightarrow(0.3+\rho(t) \cos (t),-0.2+\rho(t) \sin (t))$, where $100 \rho(t)=20+3 \sin (5 t)-2 \sin (15 t)+\sin (25 t)$. The set $E$ is the ellipse of center $(-0.3,0.1)$, with vertical major axis of length 0.3 , and horizontal minor axis of length 0.2 . The coefficients $a$ and $q$ are shown in Figure 4.1. We take $N=9$ measurements given by the different combinations of

$$
k \in 1,3,7 \text { and } \phi(x, y) \in(x, y) \mapsto x,(x, y) \mapsto y,(x, y) \mapsto 1)) .
$$

In a first experiment, we assume that the data is collected in a manner compatible with the inclusions. Namely, we use a mesh adapted to the inclusion and assume that the value at each center of mass of $E$ and $e$ is given. In Figure 4.3, we present on the 

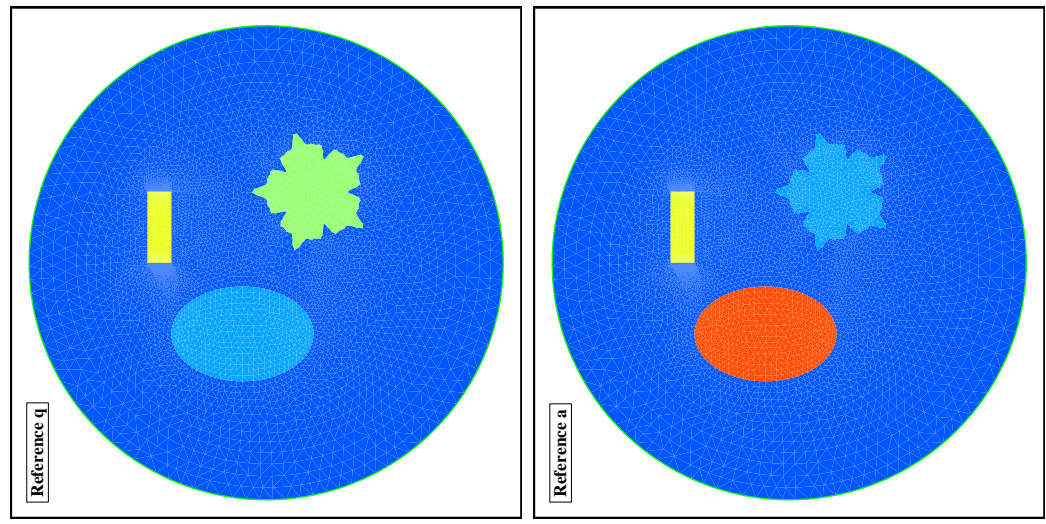

FIG. 4.1. The reference parameters.
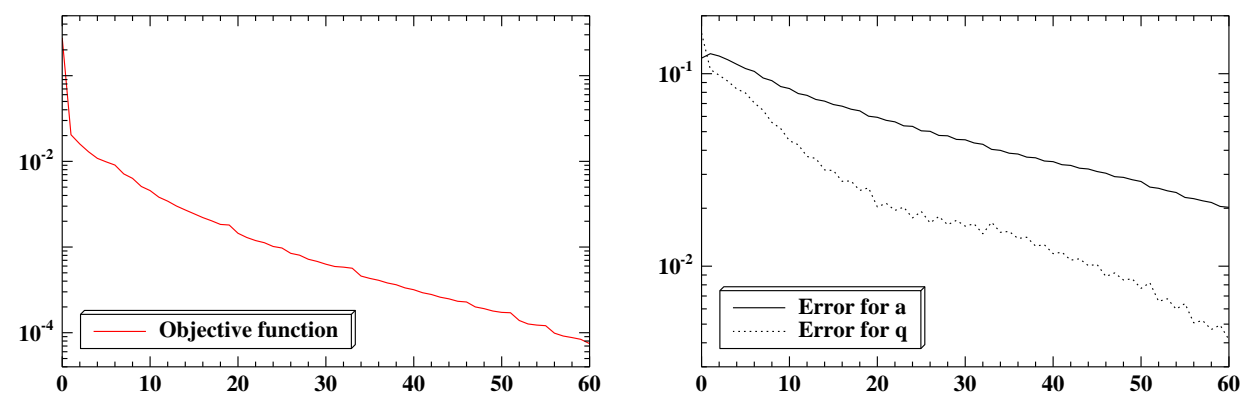

FIG. 4.2. Convergence history of the objective functional (left) and the $L^{2}(\Omega)$ errors of the parameters (right).

left $\gamma_{0}$ and $c_{0}$ used as initial guess, computed using Propositions 3.3 and 3.4. On the right, we present the final parameters as given by the gradient algorithm. In order to perform a thorough analysis, we also plot the relative error, that is,

$$
\left|\frac{a}{a^{\star}}-1\right| \text { and }\left|\frac{q}{q^{\star}}-1\right|
$$

for the initial and final parameters. The result is shown in Figure 4.4, where each levelset corresponds to an increase of the relative error of $\frac{3}{8} 10^{-2}$.

In a second experiment, we assume that the data is collected on a coarser mesh, unrelated to the location of the inclusions. The reconstruction is thus done a different mesh from the one used for synthesizing the data. The mesh used here is an uniform mesh of the disk with approximatively 1600 nodes and 3000 elements. The data is computed on the refined mesh and then projected onto the coarse mesh. Figure 4.5 shows the projection of the distributions of the reference parameters, which gives an idea of the best possible approximation. Note that the algorithm has better convergence properties with respect to $q$ than with respect to $a$. This is because the perturbation of $q$ has a lower order effect on the solution of the direct problem than the perturbation of $a$. Perturbing $a$ can dramatically affect the solution of the direct problem [8]. 

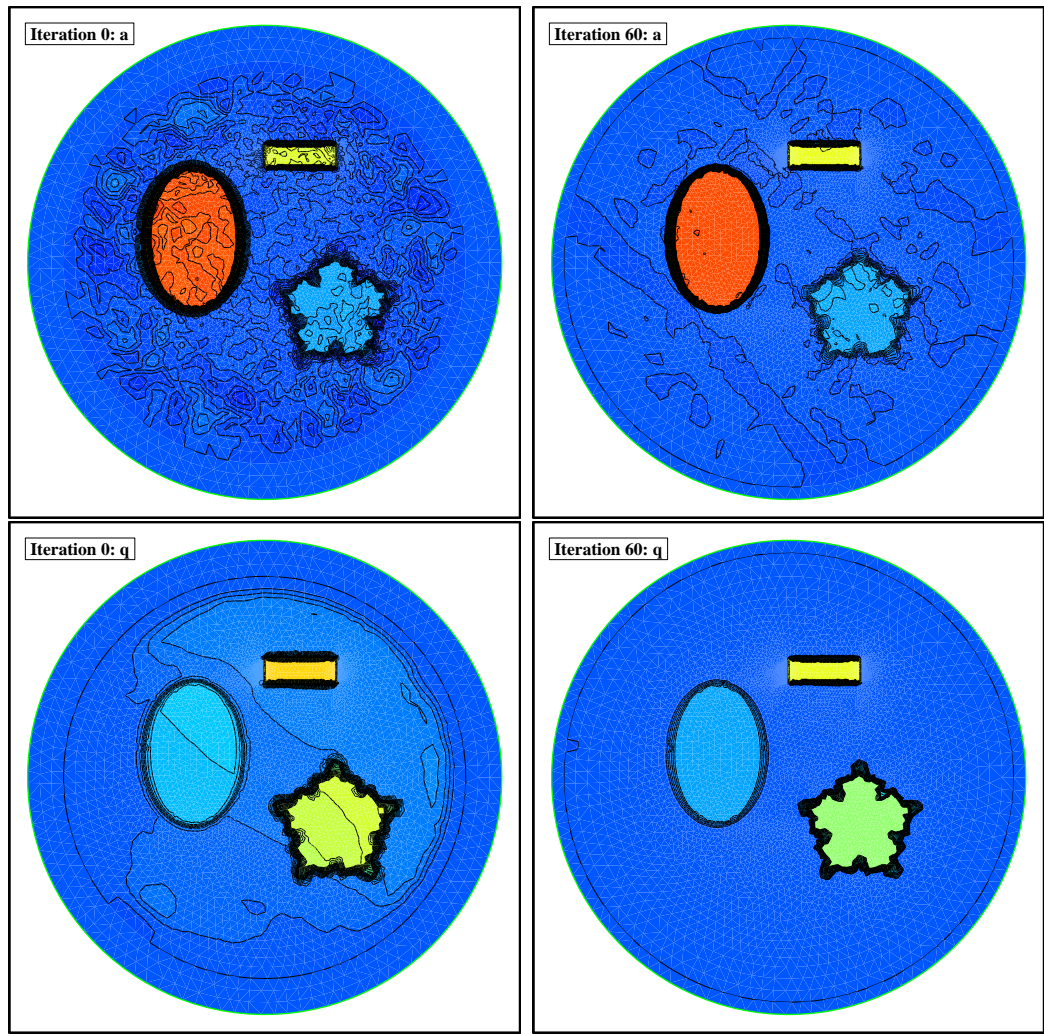

FIG. 4.3. On top, the initial (left) and final (right) a. Below, the initial (left) and final (right) $q$.

5. Conclusion. In this paper, we generalized the EIT by elastic perturbation to the microwave regime. We provided an efficient optimization algorithm to solve the reconstruction problem in two dimensions with good resolution and in a stable way. Explicit inversion formulas were used to reconstruct a good initial guess for the parameter distributions. It would be very interesting to analytically investigate the robustness, with respect to incomplete data (internal energies measured only on a part of the background domain), measurement and medium noises of the proposed algorithm. The extension of the reconstruction procedure to the full Maxwell equations in three dimensions is also challenging. Another important problem is to take into account the effects of anisotropy and dissipation (nonzero imaginary part) in the material parameters. We would also like to investigate the hybrid approach proposed in [34], where a mechanical excitation induces displacement within the object while microwave signals are measured on its boundary, since it is quite related to the model proposed here.

Acknowledgments. This work was supported by the ERC Advanced Grant Project MULTIMOD-267184. Y. Capdeboscq was supported by the EPSRC Science and Innovation award to the Oxford Centre for Nonlinear PDE (EP/E035027/1) and by the National Science Foundation under agreement No. DMS-0635607. Any opinions, findings and conclusions or recommendations expressed in this material are those of the authors and do not necessarily reflect the views of the National Science Foundation. 

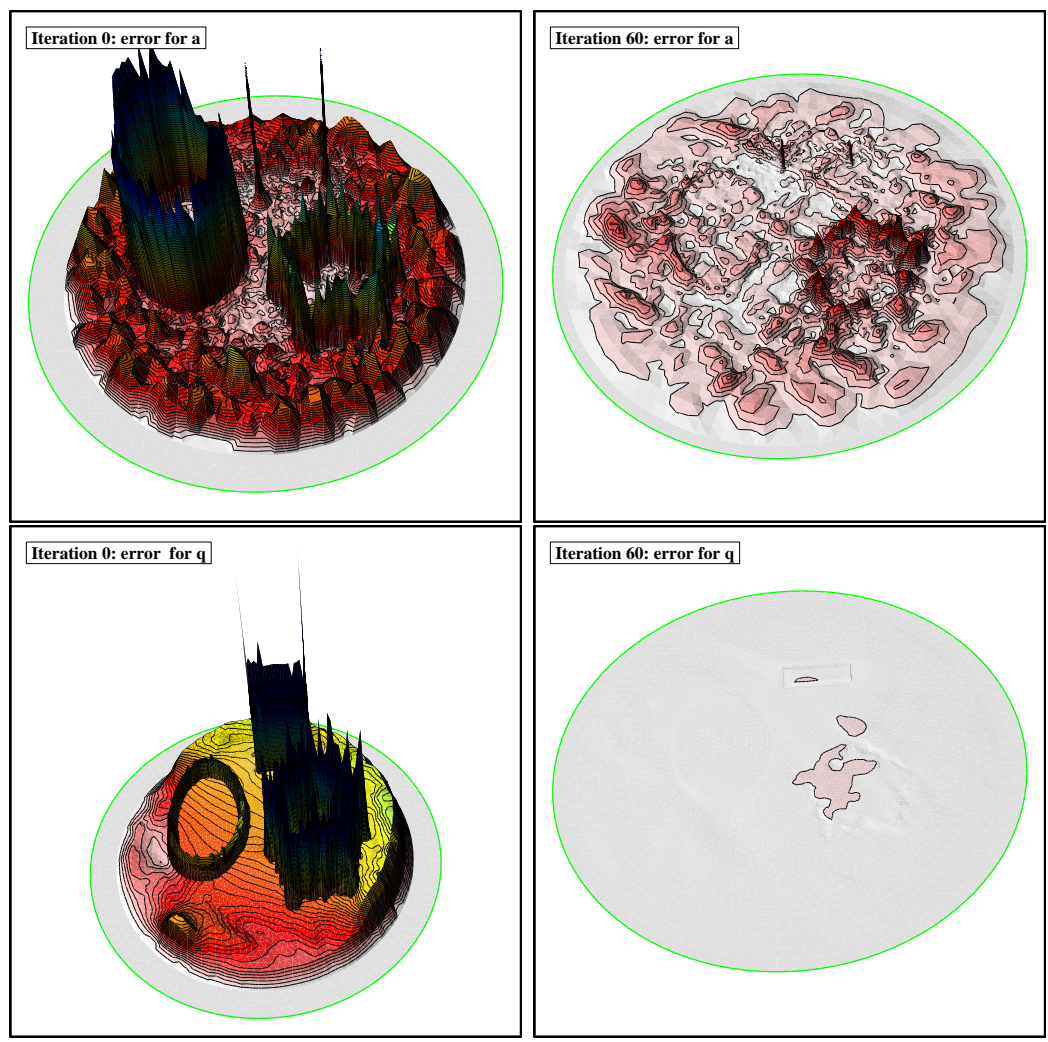

FIG. 4.4. On top, the initial (left) and final (right) relative errors on a. Below, the initial (left) and final (right) relative error on $q$.
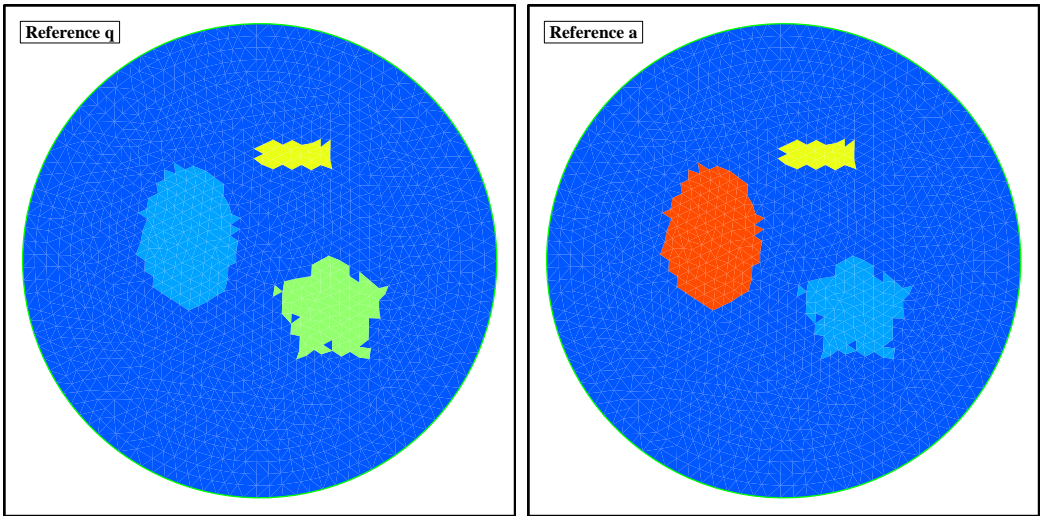

FIG. 4.5. Projection of the distributions of the reference parameters on the coarse mesh.

\section{REFERENCES}

[1] H. Ammari. An Introduction to Mathematics of Emerging Biomedical Imaging. Vol. 62, Mathematics and Applications, Springer-Verlag, Berlin, 2008.

[2] H. Ammari, E. Bonnetier, and Y. Capdeboscq. Enhanced resolution in structured media. SIAM J. Appl. Math., 70(5):1428-1452, 2009/10. 

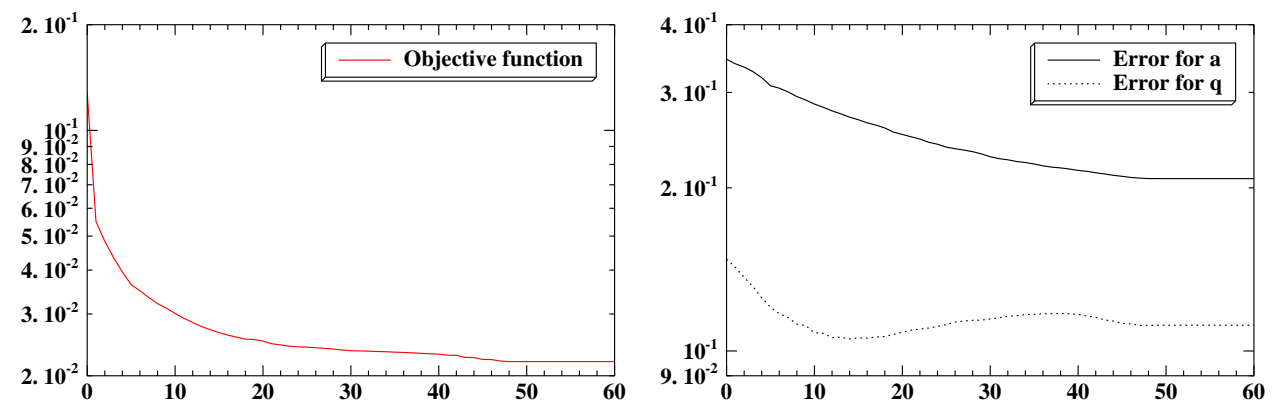

FIG. 4.6. The convergence history of the objective function (left) and the errors in $L^{2}$ norm (right) for the reconstruction on the coarse mesh.
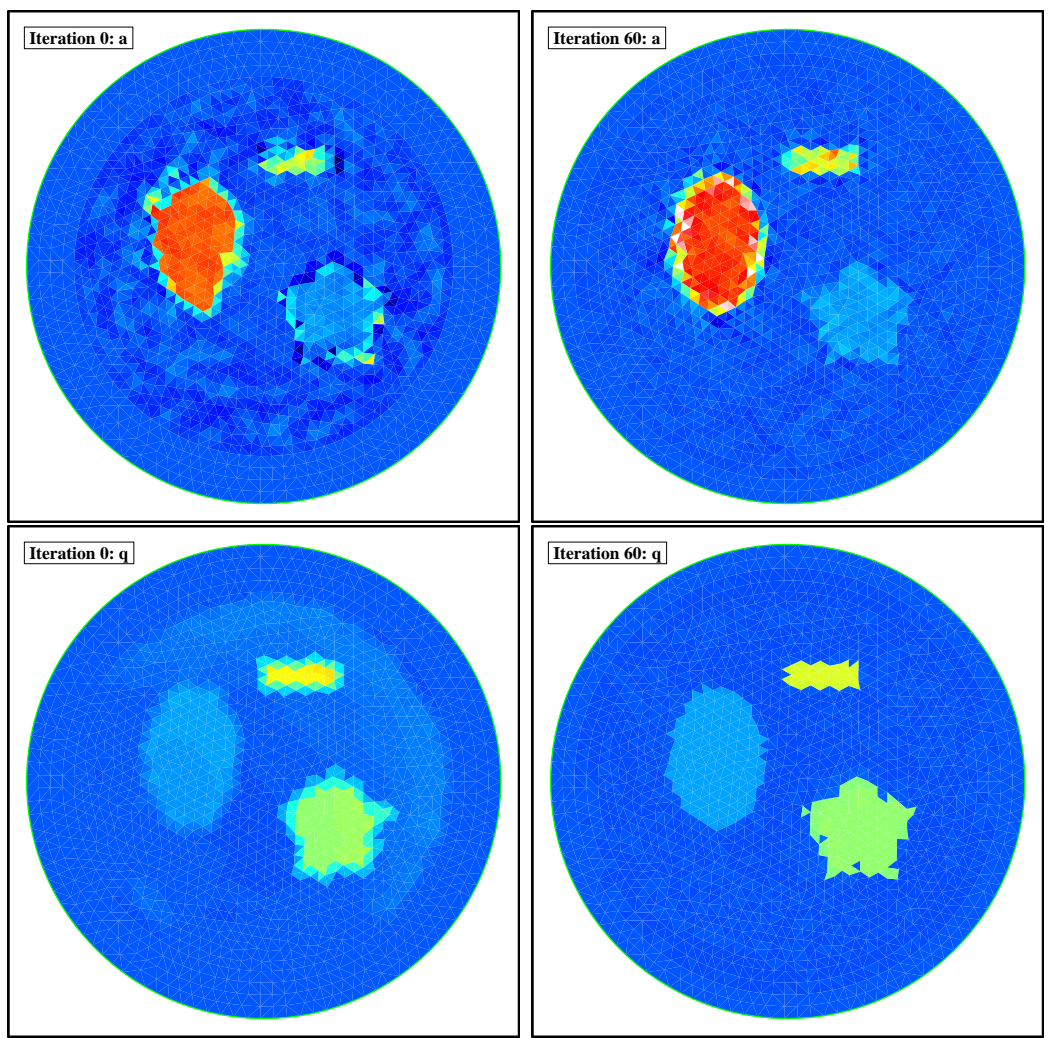

Fig. 4.7. On top, the initial and final a. Below, the initial and final $q$ for the coarse mesh.

[3] H. Ammari, E. Bonnetier, Y. Capdeboscq, M. Tanter, and M. Fink. Electrical impedance tomography by elastic deformation. SIAM J. Appl. Math., 68(6):1557-1573, 2008.

[4] H. Ammari, E. Bossy, V. Jugnon, and H. Kang. Mathematical modeling in photoacoustic imaging of small absorbers. SIAM Rev., 52(4):677-695, 2010.

[5] H. Ammari, E. Bossy, V. Jugnon, and H. Kang. Qualitative photoacoustic imaging of small absorbers. SIAM J. Appl. Math., to appear.

[6] H. Ammari, Y. Capdeboscq, H. Kang, and A. Kozhemyak. Mathematical models and reconstruction methods in magneto-acoustic imaging. European J. Appl. Math., 20(3):303-317, 2009. 

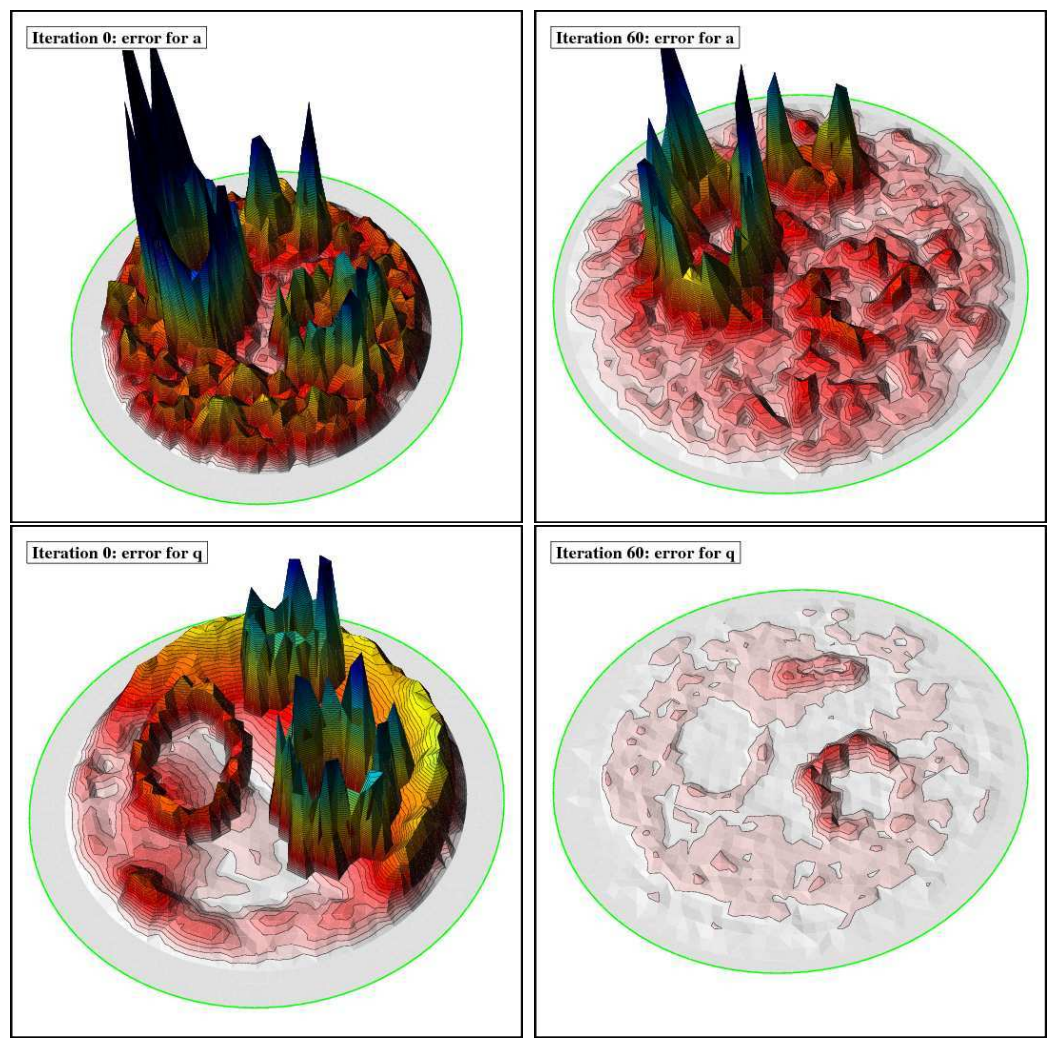

FIG. 4.8. On top, the initial and final relative errors on a. Below, the initial and final relative error on $q$ for the coarse mesh. Errors are computed with respect to the projection on the coarse mesh of the distributions of the reference parameters. Such a projection is shown in Figure 4.5.

[7] H. Ammari, P. Garapon, H. Kang, and H. Lee. A method of biological tissues elasticity reconstruction using magnetic resonance elastography measurements. Quart. Appl. Math., 66(1): 139-175, 2008.

[8] H. Ammari and H. Kang. Reconstruction of Small Inhomogeneities from Boundary Measurements, Vol. 1846, Lecture Notes in Mathematics, Springer, Berlin, 2004.

[9] H. Ammari and H. Kang. Polarization and Moment Tensors With Applications to Inverse Problems and Effective Medium Theory, Vol. 162, Applied Mathematical Sciences, Springer, New York, 2007.

[10] H. Ammari and H. Kang. Expansion Methods. Handbook of Mathematical Methods in Imaging, 447-499, Springer, New York, 2011.

[11] H. Ammari, O. Kwon, J.K. Seo, and E.J. Woo. Anomaly detection in T-scan trans-admittance imaging system. SIAM J. Appl. Math., 65, 252-266, 2004.

[12] A. Ammari, M. S. Vogelius, and D. Volkov. Asymptotic formulas for perturbations in the electromagnetic fields due to the presence of inhomogeneities of small diameter II. The full Maxwell equations. J. Math. Pures Appl., 80:769-814, 2001.

[13] K. Astala and L. Päivärinta. Calderón's inverse conductivity problem in the plane. Ann. of Math. (2), 163(1):265-299, 2006.

[14] G. Bal and J.C. Schotland. Inverse scattering and acousto-optic imaging. Phys. Rev. Letters, 104, 043902, 2010.

[15] G. Bao, S. Hou, and P. Li. Recent studies on inverse medium scattering problems. Lecture Notes in Comput. Sci. Eng., Vol. 59, 165-186, 2007.

[16] Y. Capdeboscq, J. Fehrenbach, F. de Gournay, and O. Kavian. Imaging by modification: numerical reconstruction of local conductivities from corresponding power density measurements. SIAM J. Imaging Sci., 2(4):1003-1030, 2009.

[17] F. Delbary, M. Brignone, G. Bozza, R. Aramini, and M. Piana. A visualization method for 
breast cancer detection using microwaves. SIAM J. Appl. Math., 70(7):2509-2533, 2010.

[18] D.C. Dobson. Convergence of a reconstruction method for the inverse conductivity problem. SIAM J. Appl. Math., 52(2):442-458, 1992.

[19] M. Fatemi and J.F. Greenleaf. Ultrasound stimulated vibro-acoustic spectroscopy. Science, 280:82-85, 1998.

[20] E.C. Fear and M.A. Stuchly. Microwave detection of breast cancer. IEEE Trans. Microwave Th. Tech., 48(11): 1854-1863, 2000.

[21] B. Gebauer and O. Scherzer. Impedance-acoustic tomography. SIAM J. Appl. Math., 69(2):565576, 2008.

[22] M. B. Giles and E. Süli. Adjoint methods for PDEs: a posteriori error analysis and postprocessing by duality. Acta Numer., 11:145-236, 2002.

[23] N. Irishina, O. Dorn, and M. Moscoso. A level set evolution strategy in microwave imaging for early breast cancer detection. Comput. Math. Appl., 56: 607-618, 2008.

[24] S. Kim, O. Kwon, J.K. Seo, and J.R. Yoon. On a nonlinear partial differential equation arising in magnetic resonance electrical impedance imaging. SIAM J. Math. Anal., 34, 511-526, 2002.

[25] P. Kunchment and L. Kunyansky. Mathematics of thermoacoustic tomography. Europ. J. Appl. Math., 19, 191-224, 2008.

[26] O. Kwon, J.K. Seo, and J.R. Yoon. A real-time algorithm for the location search of discontinuous conductivities with one measurement. Comm. Pure Appl. Math., 55, 1-29, 2002.

[27] Q.H. Liu, Z.Q. Zhang, T. Wang, J.A. Bryan, G. Ybarra, L.W. Nolte, and W.T. Joines. Active microwave imaging I: 2-D forward and inverse scattering methods. IEEE Trans. Microwave Th. Tech., 50(1):123-133, 2002.

[28] A. Nachman, A. Tamasan, and A. Timonov. Reconstruction of planar conductivities in subdomains from incomplete data. SIAM J. Appl. Math., 70(8):3342-3362, 2010.

[29] J.K. Seo and E.J. Woo. Magnetic resonance electrical impedance tomography (MREIT). SIAM Rev., 53(1):40-68, 2011.

[30] G. Sylvester, J. Uhlmann. A global uniqueness theorem for an inverse boundary value problem. Ann. of Math., 125:153-169, 1987.

[31] F. Triki. Uniqueness and stability for the inverse medium problem with internal data. Inverse Problems, 26(9):095014, 2010.

[32] M.S. Vogelius and D. Volkov. Asymptotic formulas for perturbations in the electromagnetic fields due to the presence of inhomogeneities. Math. Model. Numer. Anal., 34, 723-748, 2000.

[33] M. Xu and L.V. Wang. Photoacoustic imaging in biomedicine. Rev. Scient. Instrum., 77, 041101, 2006.

[34] M. Zhao, J.D. Shea, S.C. Hagness, D.W. van der Weide, B.D. Van Veen, and T. Varghese. Numerical study of microwave scattering in breast tissue via coupled dielectric and elastic contrasts. IEEE Trans. Wireless Prop. Lett., 7, 247-250, 2008. 